\title{
Bekräftigung des Anspruchs auf angemessene Behandlung und Betreuung von Menschen mit Behinderung
}

\author{
Christian Kind ${ }^{a}$, \\ Michelle Salathéb \\ a Prof. Dr. med., Vorsitzender \\ der Subkommission \\ b lic. iur., stv. Generalsekretärin \\ SAMW
}

Gemäss der Resolution 48/96 der Vereinten Nationen zur Gleichstellung von Menschen mit Behinderungen ist die angemessene medizinische Behandlung und Betreuung eine der Grundvoraussetzungen für eine gleichberechtigte Teilhabe am gesellschaftlichen Leben. Die vom Senat der SAMW am 20. Mai 2008 verabschiedeten Richtlinien «Medizinische Behandlung und Betreuung von Menschen mit Behinderung» sollen für Ärzte, Pflegende und Therapeuten in der Schweiz Unterstützung bei der Erfüllung dieser anspruchsvollen Aufgabe bieten. Neben praktischen Handlungsanweisungen finden sich im Text auch ausführliche ethische Reflexionen. Dies schien der Subkommission, die die Richtlinien ausgearbeitet hat, notwendig zu sein, um ihrem Hauptanliegen gerecht zu werden: In der medizinischen Behandlung und Betreuung von Menschen mit Behinderung muss stets der betroffene Mensch in seiner Einzigartigkeit (und nicht die Behinderung, die ihn von anderen trennt) im Zentrum stehen.

In den über 40, teilweise sehr ausführlichen Stellungnahmen zum Richtlinienentwurf wurde dieser Ansatz ausdrücklich begrüsst. Zu Missverständnissen hat mitunter die in den Richtlinien verwendete ICF-Klassifikation der WHO geführt. Gemäss dieser wird Behinderung nicht als Attribut einer Person verstanden, sondern als Resultat aus dem Wechselspiel zwischen körperlicher Schädigung, funktioneller Beeinträchtigung und sozialer Einschränkung der betroffenen Personen sowie den behindernden und fördernden Umständen und Erwartungen ihres Lebensumfelds. Dies wurde in der Überarbeitung deutlicher hervorgehoben und ein noch konsequen-

Korrespondenz:

lic. iur. Michelle Salathé

SAMW

Petersplatz 13

CH-4051 Basel

Tel. 0612699030

Fax 0612699039

mail@samw.ch

www.samw.ch
Die Richtlinien können auf der Website der SAMW (www.samw.ch) heruntergeladen oder beim Generalsekretariat der SAMW, Petersplatz 13, 4051 Basel, gratis als Broschüre bestellt werden. terer Gebrauch der ICF-Terminologie angestrebt. Nach wie vor beschränken sich die Richtlinien nicht auf bestimmte Behinderungsformen, sondern kommen in allen Fällen zur Anwendung, in denen eine Behinderung den Behandlungs- und Betreuungsprozess massgeblich prägt.

Die Richtlinien wenden sich primär an die in der medizinischen Behandlung und Betreuung involvierten Personen, d.h. Ärzte, Pflegende und Therapeuten, und sprechen deshalb meist von «Patienten mit Behinderung». Trotzdem zeigte sich bereits in der Vernehmlassung, dass sich auch Fachpersonen aus der Agogik, Heilpädagogik und Sozialpädagogik angesprochen fühlen können. Aufgrund der Bedeutung einer guten interprofessionellen Zusammenarbeit kann dies nur begrüsst werden.

Die grössten Unterschiede in den Stellungnahmen zeigten sich erwartungsgemäss bei den Themenkreisen Sexualität, Sterilisation und Elternschaft. Da sich die Stimmen, die der Betonung des Selbstbestimmungsrechts für Menschen mit kognitiver Beeinträchtigung mehr Gewicht geben wollten, und solche, denen vor allem die Verhütung von Elternschaft bei fehlender Fähigkeit, für das Wohl des Kindes zu sorgen, ein Anliegen war, in etwa die Waage hielten, beschränkte sich die Überarbeitung darauf, die Argumentation zu klären und zu Missverständnissen verleitende Formulierungen $\mathrm{zu}$ eliminieren.

Wie weitere Richtlinien der SAMW enthalten auch die vorliegenden in ihrem letzten Teil Empfehlungen, die die notwendigen Rahmenbedingungen für die Umsetzung der Richtlinien gewährleisten sollen. Die Empfehlungen richten sich deshalb an politische Instanzen und Kostenträger, an Institutionen des Gesundheitswesens und der Medizin sowie an soziale und sozialmedizinische Institutionen zur Betreuung von Menschen mit Behinderung. Die Empfehlungen erscheinen der Subkommission in Zeiten steigenden Leistungs- und Kostendrucks besonders wichtig. 